\title{
Computing a Discrete Morse Gradient From a Watershed Decomposition
}

\author{
Lidija Čomića $^{\text {a }}$ Leila De Florianic ${ }^{c}$, Federico Iuricich ${ }^{\mathrm{b}}$, Paola Magillo ${ }^{\mathrm{c}}$ \\ ${ }^{a}$ Department of Fundamental Sciences, Faculty of Technical Sciences, University of Novi Sad, Novi Sad, Serbia. \\ ${ }^{b}$ Department of Computer Science and UMIACS, University of Maryland, College Park (MD), USA. \\ ${ }^{c}$ Department of Computer Science, Bioengineering, Robotics, and Systems Engineering, University of Genova, Genova, Italy.
}

\begin{abstract}
We consider the problem of segmenting triangle meshes endowed with a discrete scalar function $f$ based on the critical points of $f$. The watershed transform induces a decomposition of the domain of function $f$ into regions of influence of its minima, called catchment basins. The discrete Morse gradient induced by $f$ allows recovering not only catchment basins but also a complete topological characterization of the function and of the shape on which it is defined through a Morse decomposition. Unfortunately, discrete Morse theory and related algorithms assume that the input scalar function has no flat areas, whereas such areas are common in real data and are easily handled by watershed algorithms. We propose here a new approach for building a discrete Morse gradient on a triangulated 3D shape endowed by a scalar function starting from the decomposition of the shape induced by the watershed transform. This allows for treating flat areas without adding noise to the data. Experimental results show that our approach has significant advantages over existing ones, which eliminate noise through perturbation: it is faster and always precise in extracting the correct number of critical elements.
\end{abstract}

Keywords: Discrete Morse theory, watershed transform, Morse-Smale complexes

\section{Introduction}

Forman's discrete Morse theory [1] is a combinatorial counterpart of Morse theory [2]. Used in shape analysis and understanding, it has been adopted for shape segmentation, and homology and persistent homology computation [3]. In particular, discrete Morse theory is the basis for an efficient and derivative-free computation of a segmentation of a discretized shape endowed with a scalar function $f$. The discrete Morse gradient fully encodes the topological structure of the function and of its domain and the regions of influence of the critical points. 35 Also, the critical net or the Morse-Smale complex can 36 be efficiently extracted from it, but the whole theory is developed under the assumption that no flat areas are present in the input data. On the other hand, flat areas are very common in real-world datasets. They can be intrinsic to a shape, like in terrains representing lakes, 41 isolines or other real flat features, or they can be due to the limited precision of acquisition devices. Currently, 43 most of the approaches based on discrete Morse theory adopt the idea of Simulation of Simplicity (SoS) [4] for eliminating flat areas (also called plateaus) by introduc- 46 ing noise into them. Both when we are analysing the 47 morphology of a scalar field, or when we are studying our domain using an existing watershed approach, subdividing the vertices of the input triangulated surface $\Sigma$ into regions of influence of the minima of the scalar function $f$ defined on $\Sigma$. Then, our algorithm proceeds in three steps. First, all the vertices in $\Sigma$, with the exception of the minima of $f$, are paired with some incident edge. This pairing is induced by the used watershed algorithm. Second, starting from the boundary of each basin, 
triangles that are contained in the basin are paired with edges. Third, the gradient obtained so far is modified to introduce critical triangles corresponding to maxima of $f$ and finally, edges and triangles between basins are paired to produce the final discrete Morse gradient. The discrete Morse gradient extends the watershed decomposition in the sense that, by navigating the gradient, we obtain the same regions of influence of the minima as the watershed decomposition. At the same time, it gives a much more powerful topological description of the function and its domain since from a discrete Morse gradient all the Morse features, including the critical net and the Morse-Smale complex, can be computed. The fact that the discrete Morse theory subsumes the watershed approach was experimentally shown in [6] by comparing their results on input data satisfying the requirement of having no flat features. Here, we go further and propose a method to complete the output of a watershed algorithm to a discrete Morse gradient. The main contri- ${ }^{100}$ butions of our work are:

- a new algorithm for computing a discrete Morse ${ }_{103}$ gradient on 2D scalar fields with flat areas,

- a constructive proof of the equivalence of the techniques based on discrete Morse theory and watershed,

- experiments showing that our algorithm is faster 108 in computing the Forman gradient compared to the 109 state of the art techniques, while it preserves the 110 flat areas.

The remainder of the paper is organized as follows. In Section 2, we review some background notions on discrete Morse theory and on the watershed transform. In Section 3, we discuss some related work on these subjects. In Sections 4, 5, 6, and 7, we describe the three steps of our algorithm. Experimental results are presented in Section 8, and concluding remarks are drawn in Section 9 .

\section{Background Notions}

We recall here some basic notions on discrete Morse ${ }_{124}$ theory [1] and on the watershed transform [7, 8], both 125 in the framework of triangle meshes. A triangle mesh ${ }_{126}$ $\Sigma$ is a set of triangles such that, for any pair of distinct ${ }_{127}$ triangles $\sigma_{1}$ and $\sigma_{2} \in \Sigma$, either $\sigma_{1}$ and $\sigma_{2}$ are disjoint, or ${ }_{128}$ their intersection is a common edge or a common vertex. ${ }_{129}$ Vertices and edges of the triangles in $\Sigma$ are also consid- 130 ered as belonging to $\Sigma$. Triangles, edges and vertices 131 are also simplexes of dimension 2,1 , and 0 , respectively. 132

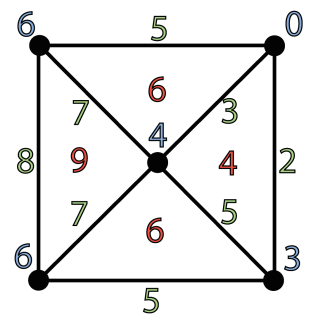

(a)

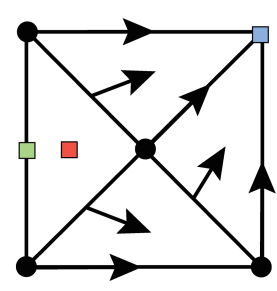

(b)
Figure 1: (a) A discrete Morse function on a triangle mesh and (b) the corresponding discrete Morse gradient. Each simplex is labeled by its function value.

A $k$-dimensional simplex, or $k$-simplex, $\sigma$ is the convex hull of $k+1$ affinely independent points. The convex hull of any subset of the vertices of $\sigma$ defines a simplex $\gamma$ which is called a face of $\sigma$, while $\sigma$ is a coface of $\gamma$. Thus, the faces of a triangle are its three edges and vertices, and the faces of an edge are its two extreme vertices. The star of a $k$-simplex $\sigma$ in $\Sigma$ is the collection of all the cofaces of $\sigma$ in $\Sigma$.

\subsection{Discrete Morse theory}

Discrete Morse theory [1] is defined for simplicial and cell complexes, but here we introduce it for triangle meshes, which are a special case of simplicial complexes. Discrete Morse theory [1] considers functions defined over all the simplexes (vertices, edges, triangles) of a triangle mesh $\Sigma$. A function $F$ defined over all the simplexes of $\Sigma$ is called a discrete Morse function, or a Forman function if, for any $k$-simplex $\sigma$, all the $(k-1)$ faces of $\sigma$ have a lower $F$ value than $\sigma$, and all the $(k+1)$-cofaces have a higher $F$ value than $\sigma$, with at most one exception. A simplex is critical if there is no such exception. Figure 1 a shows an example of a discrete Morse function $F$. Vertex 0 is critical (minimum), since $F$ has a higher value on all edges incident to it. Triangle 9 is critical (maximum), since $F$ has a lower value on all edges incident to it. Edge 8 is critical (saddle), since $F$ has a higher value on the incident triangle 9 , and lower values on its extreme vertices.

The unique exception to the above rule, which holds for a non-critical simplex, permits to pair such simplex with either one of its faces, or one of its cofaces. Thus, a discrete Morse function $F$ on a triangle mesh $\Sigma$ induces a collection of pairs $(\sigma, \tau)$, where $\sigma$ is an edge and $\tau$ is a triangle, or $\sigma$ is a vertex and $\tau$ is an edge, with $\sigma$ face of $\tau$. Such pair can be depicted as an arrow going from $\sigma$ (tail) to $\tau$ (head), as shown in Figure 1 b. An alternative way to introduce discrete Morse theory is through the notion of Forman gradient. A collection of pairs $(\sigma, \tau)$ 
defines a discrete vector field $V$ when each simplex of $\Sigma{ }_{168}$ is in at most one pair of $V$ [1].

A $V$-path is a sequence $\sigma_{1}, \tau_{1}, \sigma_{2}, \tau_{2}, \ldots, \sigma_{r}, \tau_{r}$ of $k$ - 170 simplexes $\sigma_{i}$ and $(k+1)$-simplexes $\tau_{i}, i=1, . ., r$ with 171 $r \geq 1$, such that $\left(\sigma_{i}, \tau_{i}\right) \in V, \sigma_{i+1}$ is a face of $\tau_{i}$, and ${ }_{172}$ $\sigma_{i} \neq \sigma_{i+1}$. In a triangle mesh, $V$-paths involve either 173 vertices and edges, or edges and triangles. A $V$-path 174 with $r>1$ is closed if $\sigma_{1}$ is a face of $\tau_{r}$ different from $\sigma_{r-1}$. A discrete vector field $V$ is called a discrete Morse gradient vector field (or a Forman gradient) if and only if there are no closed V-paths in $V$. A critical simplex of $V$ of index $k$ is a $k$-simplex $\gamma$ which does not appear in any pair of $V$.

There is a correspondence between discrete Morse functions and discrete Morse gradients [9]. Namely, for each such function $F$, a discrete Morse gradient $V_{F}$ can ${ }^{179}$ be constructed. Conversely, for each discrete Morse gra- 180 dient $V$ there exists a (non-unique) Forman function $F \quad 181$ such that the gradient of $F$ is $V$. The (negative) discrete 182 Morse gradient $V_{F}$ of $F$ at a vertex (edge) $\sigma$ indicates 183 the direction of a unique coface edge (triangle) $\tau$ of $\sigma,{ }_{184}$ in which $F$ is decreasing. Figure $1 \mathrm{~b}$ shows the discrete 185 Morse gradient $V_{F}$ corresponding to the Forman func- 186 tion $F$ in Figure 1 a.

As noted in [1, 10], Forman functions are hard to find, 188 and are unintuitive to work with. Thus, almost all the 189 algorithms available in the literature focus on computing 190 a discrete Morse gradient.

\subsection{The Watershed transform}

The watershed transform has been first defined for ${ }^{194}$ grey-scale images [11, 12, 7, 13, 14], and has subse- ${ }^{195}$ quently been extended to triangle meshes [15]. It is de- ${ }^{196}$ fined on an undirected labeled graph $H=\left(N_{H}, A_{H}, f\right)$, ${ }^{197}$ where the nodes in $N_{H}$, labeled by function $f$, repre- ${ }^{198}$ sent the pixels in an image, or the vertices of a triangle ${ }^{199}$ mesh, and the $\operatorname{arcs}$ in $A_{H}$ represent the edge-adjacencies ${ }^{200}$ between pairs of pixels or the edges in a triangle mesh. ${ }^{201}$ The notion of a discrete topographic distance is given in ${ }^{202}$ terms of minimum-cost paths in $H$ [7]. The lower slope ${ }^{203}$ $L S(p)$ at a node $p$ is the maximal slope linking $p$ to any ${ }^{204}$ of its neighbors of lower function value:

$L S(p)=\max \left\{\frac{f(p)-f(q)}{\operatorname{dist}(p, q)} \mid(p, q) \in A_{H}, f(q)<f(p)\right\}{ }_{208}^{207}$

where distance $\operatorname{dist}(p, q)$ is computed in domain space 210 (i.e., on the 2D plane in case of an image). If no neighbor ${ }_{211}$ $q$ exists with $f(q)<f(p)$, then $L S(p)=0$. A cost is ${ }_{212}$ associated with the arcs in $A_{H}$ defined in terms of the ${ }_{213}$ lower slope. The $\pi$-topographic distance between nodes ${ }_{214}$ $p$ and $q$ is the sum of costs of all directed arcs in path $\pi{ }_{215}$ connecting $p$ and $q$. The topographic distance $T(p, q)$ between $p$ and $q$ is the minimum of the $\pi$-topographic distances along all such paths $\pi$. The catchment basin of a minimum $m$ of $f$ is the set of nodes closer to $m$ than to any other minimum of $f$. Note that the topographic distance is actually a pseudo-distance, because it is equal to zero on distinct nodes in the same flat area.

\section{Related Work}

We provide here an overview of algorithms for extracting discrete Morse gradients, and catchment basins based on the watershed transform.

\subsection{Algorithms for discrete Morse gradient}

Algorithms based on discrete Morse theory are purely combinatorial, dimension-independent and independent of the type of the discretization of the underlying shape (domain of $f$ ).Algorithms [16, 17, 18, 19] are easily parallelizable or have been specifically developed for distributed computation. Starting from a discrete scalar function $f$ defined over the vertices of a complex $\Sigma$, they aim at constructing a discrete Morse gradient that best fits function $f$. They focus on extracting a minimum number of critical simplexes [16, 20], or they perform aposteriori simplifications to reduce their number [21, 22]. The typical applications for such algorithms are data analysis and visualization, since a discrete Morse gradient provides a computationally efficient way for extracting the regions of influence of the critical points.

The algorithm presented in [16] has been recognized as one of the most powerful. It has been adapted to triangle and tetrahedral meshes in [19, 23] and an optimized version for computing persistent homology on volumetric images has been developed in [24]. A function value is defined for each simplex by listing the field values on its vertices in lexicographic order. The algorithm processes the lower star of each vertex $v$ in $\Sigma$ independently, where the lower star of a simplex $\sigma$ is the subset of the star of $\sigma$ containing only simplexes with a lower function value than $\sigma$. Simplexes are considered in ascending order of function value and of dimension in such a way that each simplex is considered after its faces. The simplexes in the lower star of the current simplex are paired via homotopy expansion. Two simplexes, $k$-simplex $\sigma$ and $(k+1)$-simplex $\tau$, are paired when $\sigma$ has no unpaired coface and $\tau$ has only one unpaired face (i.e., $\sigma$ ). In [16] it has been proven that up to the 3D case, the critical cells identified are in one-to-one correspondence with the topological changes in the sub-level sets of the scalar function defined over the complex. 
Based on the latter, a new algorithm has been defined ${ }_{267}$ in [25] for computing a discrete Morse gradient based 268 on an input segmentation. Similarly to our proposal, the 269 input segmentation is used for guiding the gradient com- 270 putation. However, in [25] the segmentation has the pur- 271 pose of limiting the number of gradient paths between 272 adjacent regions, while in our case, as we describe in Section 4, the segmentation helps us to reconstruct the 273 correct number of critical simplexes.

\subsection{Watershed algorithms}

Several algorithms hav ture for performing the waters based on the discrete topographic distance [7], on sim- 279 ulated immersion [11, 12], or on rain falling simulation 280 [15, 14].

The approach based on topographic distance directly 282 applies the definition of catchment basins. The image in- 283 tegration algorithm by Meyer [26, 7] is a variation of the 284 Dijkstra-Moore algorithm [27] for computing the short- 285 est path, in terms of topographic distance, from a source 286 node to every other node in a graph. The hill climbing 287 algorithm [7] is a simplified version of the image integra- 288 tion approach, which applies to regular grids, since the 289 distance between two adjacent nodes $p$ and $q$ in domain 290 space is assumed to be constant.

The intuitive idea behind the simulated immersion ap- 292 proach [11, 12] is that of letting water raise from minima. 293 When applied to the graph $H$ describing the image or the 294 triangle mesh, the algorithm expands catchment basins 295 by processing the nodes of $H$ by increasing function val- 296 ues. When a certain level $h$ is reached, all catchment 297 basins of minima with value $h^{\prime}<h$ have been started 298 and contain just nodes with function values lower than $h$. 299 Processing level $h$ will add new nodes to existing basins, 300 and will start new basins from minima having a function 301 value equal to $h$. The expansion of existing basins treats 302 flat areas in a transparent way.

The watershed approaches discussed so far have in common the idea of growing catchment basins upwards from the minima of $f$. The rain falling paradigm [15, 14] uses the idea of letting water fall down from 305 each vertex until it reaches a minimum. The algorithm 306 descends from each node to its lowest adjacent node 307 until a minimum is reached. The algorithm in [15] is 308 for triangle meshes, while the one in [14] is for regular 309 grids. Both of them allow for the treatment of flat areas. 310 In [15], all such areas are found in a preliminary step 311 and each of them is treated as a single node. In [14], flat 312 areas are found as they are encountered during the de- 313 scent. An implementation of the rain falling simulation 314 for triangle meshes has been used in [28].
In the output of watershed algorithms, not all nodes belong to a catchment basin. In the approaches based on topographic distance and on simulated immersion, nodes that have the same distance from different minima, or are reached by water from different minima, are labelled as belonging to watershed lines (watershed nodes).

\subsection{Removal of Flat Areas}

Simulation of Simplicity (SoS) [4] is the most widely used method to resolve differentiability in topological analysis in the discrete case. Given a complex with flat areas, a strictly increasing ordering of vertices is defined. The new indexes preserve the old order (induced by function values) for distinct value data and impose a new ordering for vertices having the same value. The main drawback of SoS is that it introduces spurious critical points that are not present in the original data. To mitigate this problem an improved version of the SoS approach has been introduced in [22]. Two sorted queues are used for imposing a breadth first visit of the flat areas. In this manner, no spurious minima are created but saddles and maxima are still introduced without control. The algorithm presented in [28] is the only one, to the best of our knowledge, that address the problem of perturbing data in a such a way that the modified scalar field does not have missing or extra critical points. This algorithm works on triangle meshes only. It iteratively addresses vertices on the boundary of a flat area and slightly raises or lowers them (according to specific rules), in order to progressively "erode" each plateau without creating new maxima or minima, or deleting existing ones. The method has to use a priority queue of candidate vertices for the various rules, as each modified vertex creates new candidates. Moreover, in order to avoid machine precision errors, it applies a symbolic modification of height values followed by a final rescaling of all the vertices. For such reasons, it is rather complicated and intrinsically slower than using perturbation.

\section{Computing the discrete Morse gradient}

In this section, we describe our approach, that combines watershed transform and discrete Morse theory, for computing a Forman gradient from a scalar field. The input of our algorithm is a triangle mesh $\Sigma$ endowed with a scalar function $f$ at its vertices, together with a partition of the vertices of $\Sigma$, produced by a watershed algorithm (see Section 3). In our implementation we have used simulated immersion, but any other watershed algorithm can be used as well.

We apply the watershed algorithm to the graph $H$ formed by the vertices and edges of $\Sigma$, where each node 


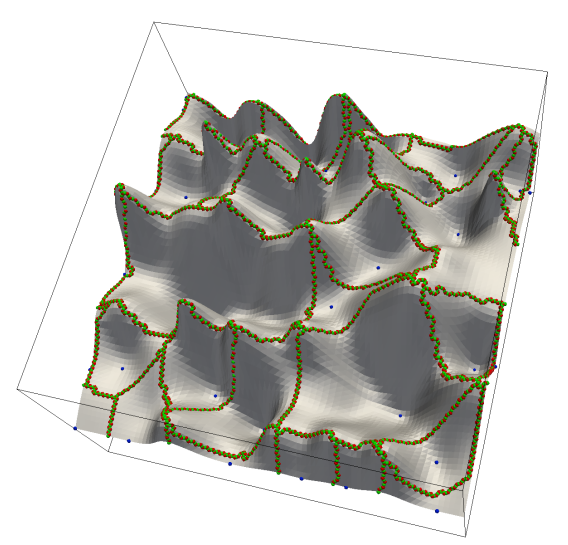

Figure 2: A triangle mesh representing a terrain, where scalar function $f$ is elevation. Dots indicate the unpaired or critical simplexes after step 2. All triangles contained in some basin are paired, while triangles on the boundary of two or more basins are still unpaired.

of $H$, which is a vertex of $\Sigma$, is labeled with the corresponding value of $f$. Recall that the watershed algorithm applied to $H$ labels the nodes of $H$ as belonging to catchment basins or to watershed lines. We remove watershed nodes by assigning them to a basin in the following way. A queue of watershed nodes having a lower adjacent node assigned to some basin is built. At each step, a watershed node $v$ is extracted from the queue, labeled as belonging to the same basin as its neighbor with lowest function value, and watershed nodes adjacent to $v$ are inserted into the queue. The process continues until the queue is emptied. The result of this preprocessing is a partition of the vertices of $\Sigma$ into catchments basins.

The discrete Morse gradient $V$ induced by the watershed algorithm is built in three steps:

1. Vertex-edge pairing. A discrete Morse gradient $V^{\prime}{ }_{353}$ is constructed having only vertex-edge pairs, such 354 that each vertex, which is not a minimum, is paired 355 in $V^{\prime}$.

2. Edge-triangle pairing inside basins. $V^{\prime}$ is extended to $V^{\prime \prime}$ by adding edge-triangle pairs in such a way that each triangle lying completely inside some basin (i.e., such that all three vertices belong to the basin) is paired (see Figure 2).

3. Edge-triangle pairing between adjacent basins. The ${ }^{363}$ final discrete Morse gradient $V$ is built from $V^{\prime \prime}{ }^{364}$ by determining critical triangles and pairing the re- ${ }^{365}$ maining edges and (non-critical) triangles whose ${ }^{366}$ vertices belong to different basins.

In the following three sections, we describe the three 369 steps in detail, and prove the correctness of each step, 370

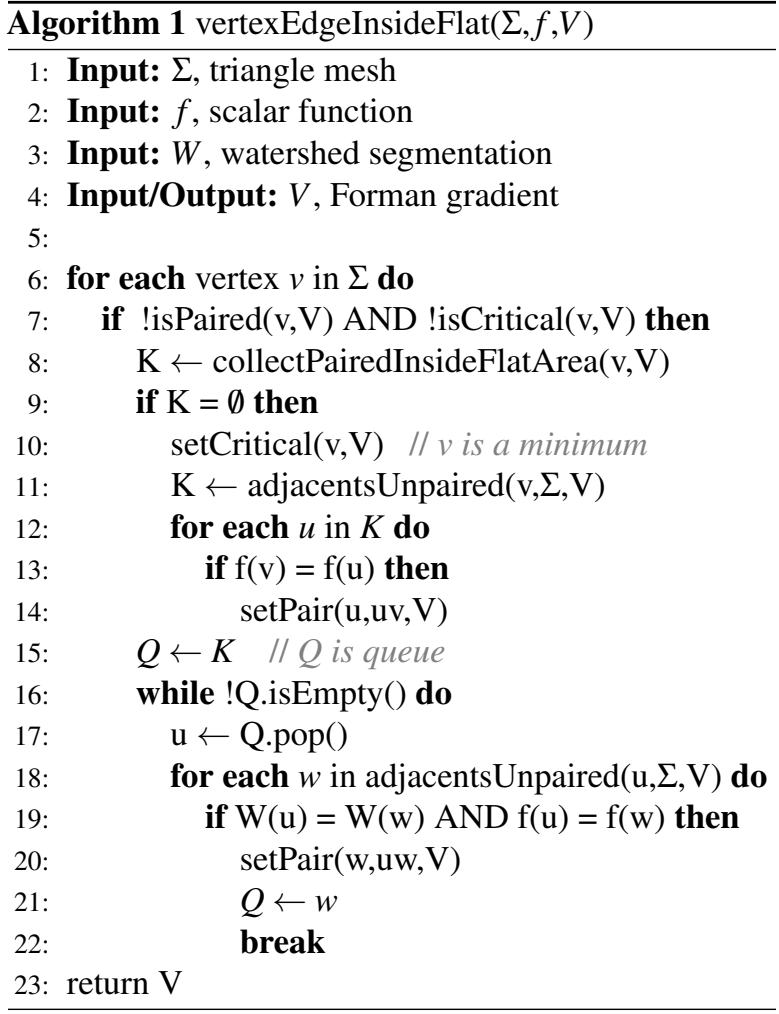

and thus of the whole algorithm. We show that the pairing of cells established by our algorithm defines a discrete Morse gradient $V$ on $\Sigma$ such that there is a one-toone correspondence between the critical points of the scalar function $f$ and the critical simplexes of $V$.

\section{Step 1: Vertex-Edge Pairing}

We first pair the vertices that do not belong to any flat area. We construct the vertex-edge pairs directly from the watershed partition. Each vertex $v$ in $\Sigma$ is paired with its lowest adjacent vertex $u$, belonging to the same basin, only if they do not share a flat edge. A second sweep on the vertices of $\Sigma$ is used for pairing vertices inside flat areas (see Algorithm 1). These are exactly those left unpaired after the first sweep (row 7). For each unpaired vertex we recursively collect the adjacent vertices inside the flat area. Among them we select those that have been already paired $(K \leftarrow$ collectPairedInsideFlatArea $(\cdot))$. If $K=\emptyset$ the flat area is a minimum and $v$ is set as critical (see Figure 3 3 ). Each unpaired vertex $u$, adjacent to $v$, is then paired with $v$ if the edge $u v$ is flat (rows 11 to 14 ). After that, plateau minima and non-minima are treated in the same fashion. We insert the vertices, belonging to the plateau, that have been already paired, in a queue $Q$ (row 17). Vertices in $Q$ are recursively 


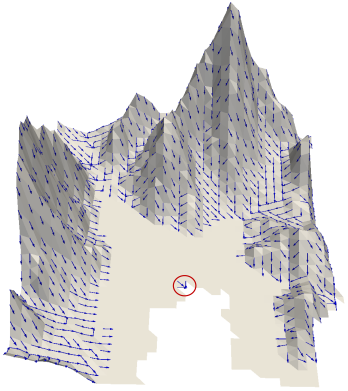

(a)

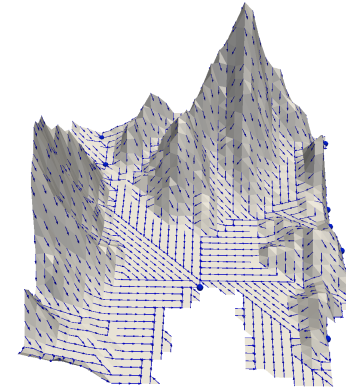

(b)
Figure 3: Pairings inside a plateau minimum. (a) A vertex is chosen as representative minimum (inside the red circle) and all its adjacent vertices are paired. (b) The paths are then extended starting from those vertices until all the vertices in the plateau are paired.

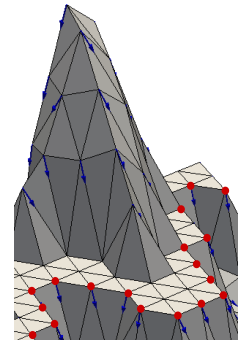

(a)

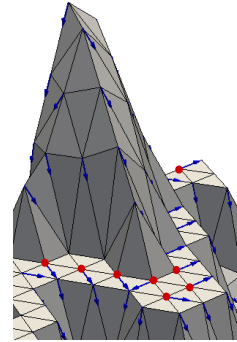

(b)

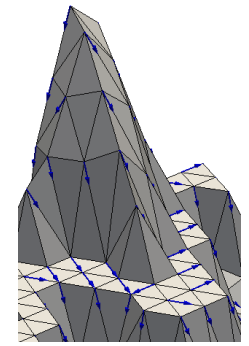

(c)
Figure 4: Pairings inside a non-minimum plateau. (a) Starting from the vertices belonging to the plateau and already paired (red dots), the 4 other vertices are progressively paired (b), (c).

paired, in a breadth first manner, with adjacent vertices having the same value of $f$ and belonging to the same basin (row 20). The latter corresponds to extending the gradient paths upwards, starting from vertices having gradient paths that exit from the plateau (see Figure 47, or from the artificial minimum (see Figure $3 \mathrm{p}$ ) in case of a plateau minimum.

\subsection{Correctness of step 1}

We have to show that the constructed set of vertex- 426 edge pairs, that we denote with $V^{\prime}$ : (i) has no cycles; (ii) 427 all vertices are paired, with the exception of the minima 428 of $f$, and of one artificial minimum for each plateau ${ }_{429}$ minimum.

We observe that the input of our algorithm is a correct 431 watershed output. Distinct minima of function $f$ (includ- 432 ing plateau minima) have different labels, and each other 433 vertex takes its basin label from one of its lower adjacent 434 vertices.

Properties (i) and (ii) are trivially satisfied for non- 436 flat edges. For flat edges, let $P$ be the connected set of ${ }_{437}$ vertices that form a plateau, let $A$ be the set of edges in $\Sigma$ that connect vertices in $P$ and let $A^{*}$ be the set of edges in $A$ that are paired with a vertex in $P$. The graph $\left(P, A^{*}\right)$ is a spanning forest of the graph $(P, A)$. The roots of trees in $\left(P, A^{*}\right)$ are vertices paired with an edge connecting it to a lower neighbor (for non-minimum plateaus) or the artificial minimum (for plateau minima). Since there are no cycles in a forest, property (i) is satisfied. Since $(P, A)$ is a connected graph, each node in $P$ is either a node or a root of some tree, implying property (ii).

\section{Step 2: Edge-Triangle Pairing Inside Basins}

We extend the collection $V^{\prime}$ of vertex-edge pairs produced at step 1 by adding edge-triangle pairs to $V^{\prime}$ for each triangle $t$ where the three vertices of $t$ belong to the same basin $R$. In that case, for short, we say that triangle $t$ is inside $R$. We denoted the extended set as $V^{\prime \prime}$.

We use a priority queue $Q$ of edge-triangle pairs organized in descending order of the interpolated function value at the centroids of the edges. $Q$ is initialized with pairs $(e, t)$, where $e$ is an unpaired edge on the boundary of $R$ and $t$ is an unpaired triangle inside $R$ and incident in $e$. Then, for each edge-triangle pair $(e, t)$ in $Q$, if $t$ is already paired the pair is skipped. Note that the pairing of $t$ might have occurred while processing another edge of $t$ with higher priority than $e$. Otherwise, edge $e$ and triangle $t$ are paired in $V^{\prime \prime}$. Each unpaired edge $e^{\prime}$ of $t$, with $e^{\prime} \neq e$, is added to $Q$ together with the triangle $t^{\prime}$ sharing $e^{\prime}$ with $t$ only if $t^{\prime}$ is inside the same basin as $t$. The process continues until $Q$ becomes empty.

The above process progressively erodes the set of unpaired triangles inside a basin, starting from triangles lying on the boundary of the set (which are paired first) and then moving into the basin.

\subsection{Correctness of step 2}

We have to show that (i) the edge-triangle pairs constructed do not form cycles, and (ii) all triangles lying inside a basin have been paired.

Let us consider the set of triangles inside a basin $R$. This set consists of one or more connected components, each bounded by one or more cycles of edges.

$Q$ is initialized using the unpaired edges belonging to such cycles. Each time a triangle $t$ is paired with one of those edges, the other (unpaired) edges of $t$ are inserted in the queue. Thus, after each pairing, we obtain a new set of bounding cycles, which are contained in the previous ones, until there are no more unpaired triangles inside $R$. Property (i) is shown by noting that generated 


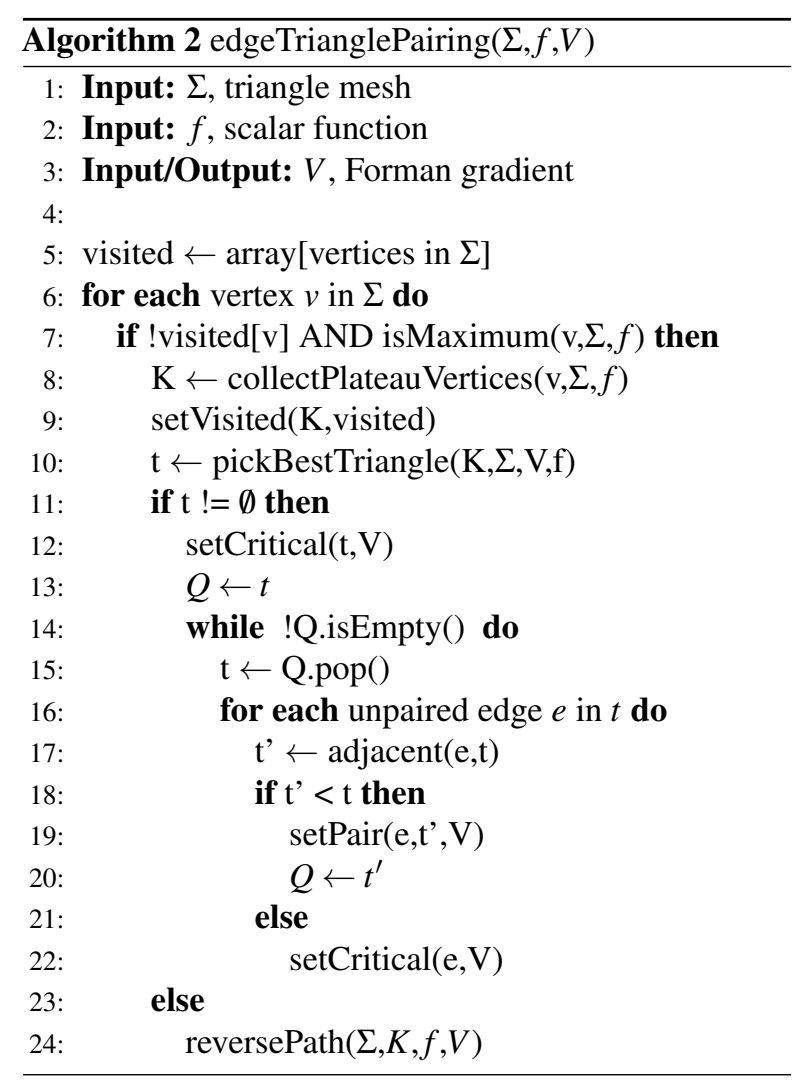

gradient arrows are always directed from outer bound- 466 ary (before the update) to inner boundary (after the up- 467 date). Figure 5 shows a working example; note that a ${ }_{468}$ connected component can be split into two, and in that ${ }_{469}$ case each of the two cycles has an edge added to the 470 queue.

For property (ii), we have to show that $Q$ is initialized 472 with at least one unpaired boundary edge for each con- 473 nected component of (unpaired) triangles inside a basin 474 $R$. This follows from the fact that there is, at least, one 475 unpaired edge $e$ in each cycle $C$ bounding a connected 476 component of triangles inside a basin $R$ in the initial con- 477 figuration. Otherwise, there would be a vertex in $C$ that 478 is paired with more than one edge, or there would be 479 a closed path in $V^{\prime}$ consisting of vertices and edges in 480 cycle $C$, which is false (as we proved in Section 5.1).

\section{Step 3: Edge-Triangle Pairing on Basin Bound- ${ }^{48}$ aries}

At the end of the second step, all triangles inside a 486 basin are paired. Unpaired triangles are limited to those ${ }_{487}$ on the boundary between basins, and are organized into 488 triangle strips, as depicted in Figure 2 (general view) and 489 in Figure 6a (detail).

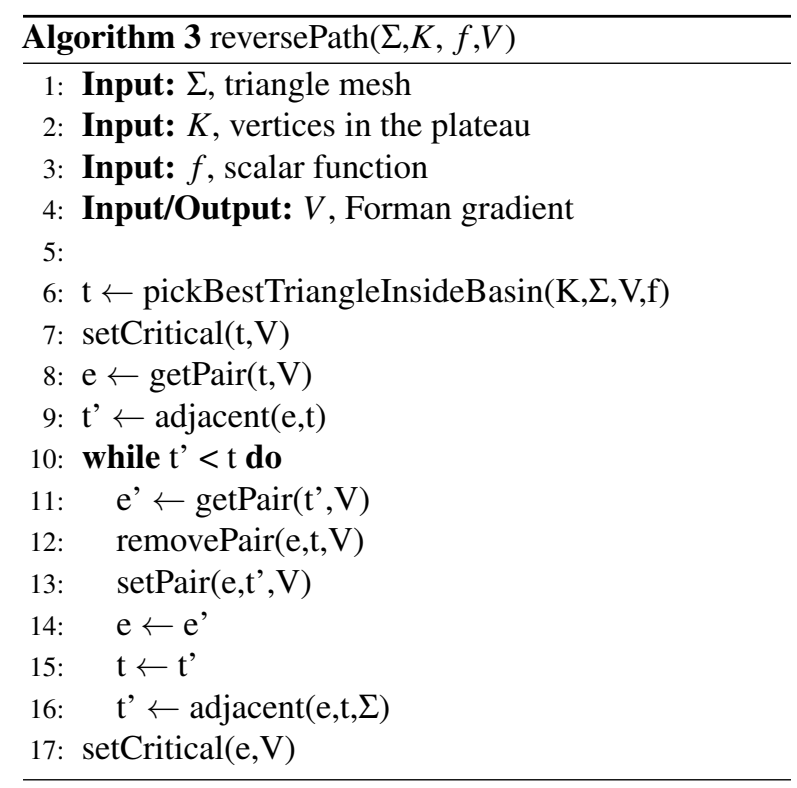

The following step is described in Algorithm 2 We identify both isolated maxima of $f$ (vertices of $\Sigma$ having all the adjacent vertices with a lower function value), and plateau maxima, which are sets of connected vertices such that all the vertices adjacent to them have the same or a lower function value. For each maximum, we pick the corresponding critical triangle (row 10) among those incident in the maximum vertex, or in one of the vertices of the plateau maximum. Precisely, the critical triangle is the unpaired triangle having the maximum number of unpaired edges on its boundary. If two or more triangles have the same number of unpaired edges, we pick the one having the centroid with the highest (interpolated) function value. From maximum triangles, we start a descending process in which we create new gradient pairs. We say that $t^{\prime}<t$ when the the third vertex of $t^{\prime}$ (not in $t$ ) has a lower function value than the two shared vertices. Let $t$ be the current triangle (initially, a maximum triangle). We initiate a descending gradient path from each of its unpaired edges (row 16). We pair each of such edges $e$ with its incident triangle $t^{\prime}$ different from $t$, if $t^{\prime}<t$. Otherwise, we declare edge $e$ as critical. A working example is shown in Figure 6 Cases exist where maxima lie inside some basin $R$ instead of lying on its boundary. In this case the identified critical maximum is surrounded by paired triangles. In such cases (see Algorithm 3), triangle $t$ is chosen based on the function value of the centroid and declared critical (rows 6 and 7). Then, the gradient path originally converging to $t$ is reversed. 


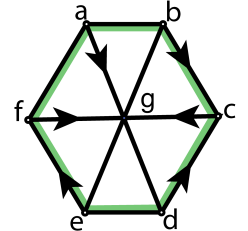

(a)

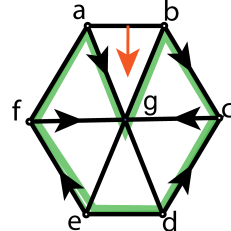

(b)

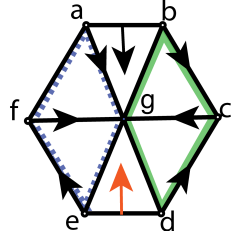

(c)

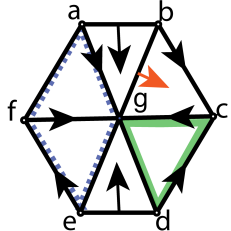

(d)

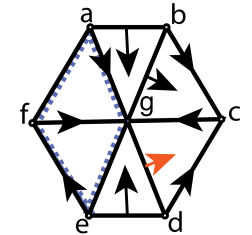

(e)

Figure 5: Successive pairings inside a basin and updates of the corresponding cycles. (a) Discrete Morse gradient $V^{\prime}$ with only vertex-edge pairs. Vertices $a b c d e f$ define edges in cycle $C$. (b) After pairing of unpaired edge $a b$ with triangle $a b g$, edge $a b$ is replaced with edges $a g$ and $b g$ in cycle $C$. (c) After pairing of unpaired edge $d e$ with triangle $d e g$, cycle $C$ is split in two, $C^{\prime}$ defined by bcdg and $C^{\prime \prime}$ by age $f$. (d) After pairing of unpaired edge $b g$ with triangle $b c g$, cycle $C^{\prime}$ surrounds a unique triangle $c d g$, (e) which is then paired with unpaired edge $d g$.

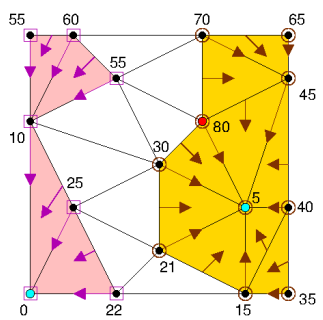

(a)

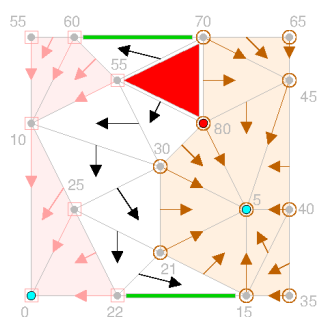

(b)

Figure 6: A terrain with two minima (cyan) and one maximum (red). (a) Pairs obtained after the first two steps. (b) The red triangle is defined as a maximum and the descending paths from its edges to the two green critical edges are created.

\subsection{Special cases}

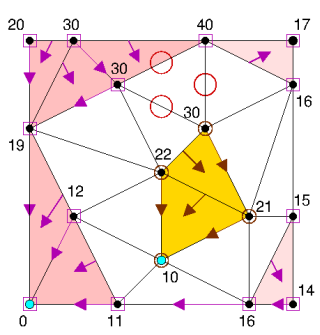

(a)

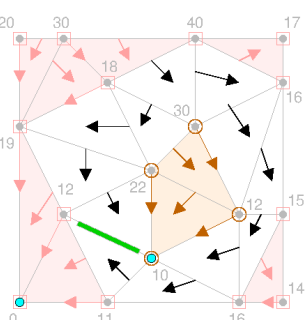

(b)

Figure 8: (a) A basin (yellow) completely contained in another one (pink). The descent starts from the unpaired triangle having three unpaired edges (marked with red circles). (b) The set of triangles paired with edges and the new critical (green) edge.

The above process may leave unpaired triangles and 501 edges when the domain has a boundary (see Figure $7 \mathrm{~h}$ ) 502 or when a basin $R_{1}$ is completely contained in another 503 basin $R_{2}$ (see Figure 8 a).

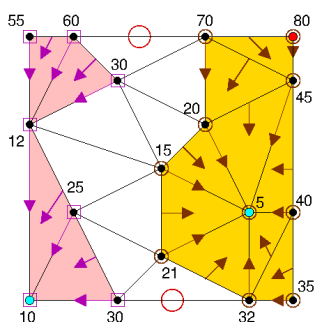

(a)

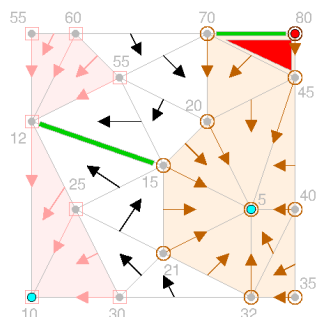

(b) of $R_{2}$ (it has endpoints in $R_{2}$, and the third vertex of the unpaired triangle $t$ incident to $e$ is in $R_{1}$ ), or there is a critical edge $c$ with endpoints in $R_{2}$, such that there is a gradient $V^{\prime \prime}$-path starting from an edge $e$ on the boundary of $R_{2}$ and ending at a triangle incident to $c$. In the latter case, we reverse this gradient path making $e$ unpaired. Among such candidates, we select the one with the highest interpolated function value on the unpaired edge $e$, then we pair $e$ and $t$, and we restart a descent from each other unpaired edge of $t$ (as described before). This process continues until all triangles have been paired. An example involving a boundary edge is shown in Figure 7 An example with a basin contained in another basin is shown in Figure 8

Figure 7: A terrain with two minima (cyan) and one maximum (red). (a) Pairs obtained after the first two steps. (b) The red triangle is 515 defined as a maximum and the descent from it ends at the green critical edge on the boundary, white triangles remain unpaired. The descent is restarted from the boundary triangles marked with circle ending on the other green (critical) edge.

In such cases, we need to find other triangles (and ${ }_{520}$ edges) to restart a descending path. In the first case, an 521 unpaired triangle $t$ must be adjacent to the boundary of ${ }_{522}$ the domain along an unpaired edge $e$. In the second ${ }_{523}$

\subsection{Correctness of step 3}

Step 3 creates one maximum triangle in the gradient field for each maximum of function $f$. We have to show that no cycles are created during the descent. Since all the gradient paths are built following a descent on the function value, no closed V-paths can be obtained.

In the special cases, we have to show that at least one triangle-edge pair exists as a candidate to restart the descent. In case where an unpaired triangle is on the 
boundary of the domain, the boundary edge must be un- 574 paired, because it could only be paired with the triangle 575 itself. In case where one basin $R_{1}$ is completely con- 576 tained in another basin $R_{2}$, if an unpaired edge $e$ exists 577 on the boundary of $R_{2}$ that is incident to an unpaired 578 triangle $t$ in the triangle strip between $R_{1}$ and $R_{2}$, we are ${ }_{579}$ done. Otherwise, let us consider the simplicial complex 580 $\Sigma_{R}$ defined by connected component of triangles in $R_{2} 581$ that encloses $R_{1}$ and all the incident edges and vertices, 582 together with the restriction $V_{R}^{\prime \prime}$ of $V^{\prime \prime}$ to $\Sigma_{R}$. Note that ${ }_{583}$ each two vertices in $\Sigma_{R}$ are connected through a $V_{R}^{\prime \prime}$-path. 584 Since $\Sigma_{R}$ has a hole corresponding to $R_{1}$ and $V_{R}^{\prime \prime}$ is a dis- 585 crete Morse gradient, there must be at least one critical 586 edge $c$ in $\Sigma_{R}$ incident to two triangles $t_{1}$ and $t_{2}$ in $\Sigma_{R}$. 587 Since all triangles in $\Sigma_{R}$ are paired, there is a gradient 588 $V_{R}^{\prime \prime}$-path $p_{1}$ starting at an edge $e_{1}$ on the boundary of $R_{2} \quad 589$ and ending at $t_{1}$, and similarly for $t_{2}$. Such gradient paths 590 define triangle strips, i.e., each two consecutive triangles 591 in the path share an edge that is paired with one of the 592 two triangles. The two edges $e_{1}$ and $e_{2}$ must be on two 593 different cycles of boundary edges of $R_{2}$, because other- 594 wise the union of triangles in the two paths $p_{1}$ and $p_{2} 595$ would disconnect the vertices in $\Sigma_{R}$, i.e., the endpoints 596 of $c$ would not be connected through a gradient path in 597 $\Sigma_{R}$. For similar reasons, such gradient paths for two dif- 598 ferent critical edges cannot start at edges belonging to 599 the same two cycles. Thus, for each basin $R_{1}$ enclosed 600 in $R_{2}$ there must be an edge $e$ on the boundary of $R_{2} 601$ that is incident to a triangle $t$ with the third vertex in 602 $R_{1}$, such that there is a gradient $V^{\prime \prime}$-path starting at $e$ and 603 ending at some triangle in $R_{2}$ incident to a critical edge $c$. 604 The reversal of this gradient path will pair $e$ with $t$, and 605 step 3 of the algorithm can proceed from the remaining 606 two edges of $t$. From the discussion above, it follows 607 that path reversal and the pairing of triangles between 608 $R_{1}$ and $R_{2}$ will not create closed gradient $V$-paths. Thus, 609 the gradient field $V$, built by our algorithm, is a discrete 610 Morse gradient on $\Sigma$ : it is defined by vertex-edge and 611 edge-triangle pairs, each element of $\Sigma$ is in at most one 612 pair and there are no closed $V$-paths.

\section{Results and discussion}

The presented approach is based on the idea of in- 617 troducing a single critical vertex or triangle wherever 618 there is a minimum or maximum in the dataset in the 619 piecewise-linear sense (see [29]). We have proved that 620 no vertices and triangles (other than those associated 621 with minima and maxima, respectively) remain unpaired 622 after applying our algorithm and, thus, the number of 623 critical minima and maxima is correct. We checked that 624 also the number of critical edges found is always correct 625 by exploiting the Euler formula and the known Euler characteristic of the domain of our test meshes.

In this section we compare the results obtained using our approach with those combining Simulation of Simplificty (SoS) [4], or its improved version $(\mathrm{SoS}++)$ [22], with the algorithm for Forman gradient computation based on homotopy expansion in [16]. For encoding the triangle mesh and representing the Forman gradient we are using the efficient representation described in [19]. Experiments have been performed on a MacBook Pro with a $2.8 \mathrm{GHz}$ processor and $16 \mathrm{~GB}$ of memory. The segmentation algorithm used in our experiments is the watershed by simulated immersion [11].

Tests have been performed on a variety of triangle meshes representing both terrain datasets and 3D shapes (closed triangulated surfaces). The results are shown in Table 1. For each dataset, we present the number of vertices $(|V|)$, triangles $(|T|)$ and the total number of critical points $(|C P|)$ of the scalar field. Note that this latter is equal to the total number of critical simplexes of the discrete Morse gradient extracted by our algorithm. The timings required by the new algorithm are shown separately for each step. We can notice that the time required by step 1 is generally less than that required by step 2 , since steps 1 and 2 cycle over vertices and triangles, respectively, and there are twice as many triangles as vertices in any triangle mesh. For the same reason, step 3 requires about the same time as step 2 since it cycles over the triangles for a second time. Column Tot indicates the time required by the algorithm including also the computation of the watershed segmentation. Columns SoS and SoS++ in Table 1 show the number of critical points obtained imposing a total order on the vertices based on the respective methods. We can notice the drawback of introducing noise for perturbing the input function around flat areas. When the input function has large flat areas, the number of spurious critical simplexes found by the algorithm increases up to a maximum of $46 \%$ of the total number of critical simplexes found by our algorithm. SoS++ solves only partially the problem and for some datasets more than $40 \%$ of the critical simplices identified are still spurious. Figure 9 shows this behavior on three toy expamples; Figures 10 and 11 show it on two real datasets. On the left, we show the critical simplexes extracted by our algorithm, on the right those extracted by the algorithm in [16] after SoS is applied. The last column presents the time required for computing the Forman gradient using the algorithm described in [16]. Notice that the latter does not include the time required for creating the total order with SoS or SoS++. By comparing the timings of the two algorithms, we see that our method takes less time in general, being 


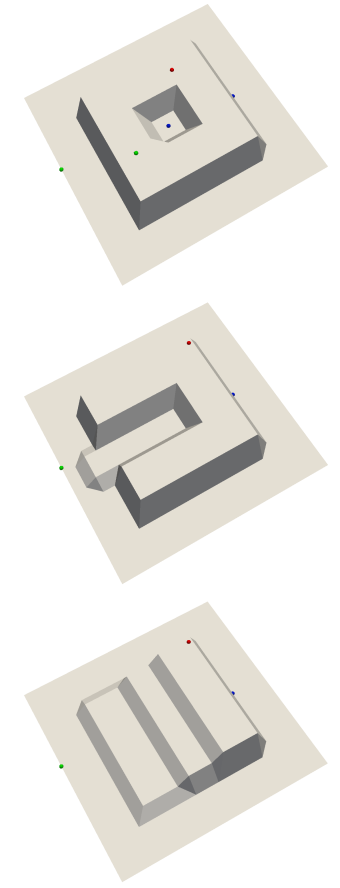

(a)

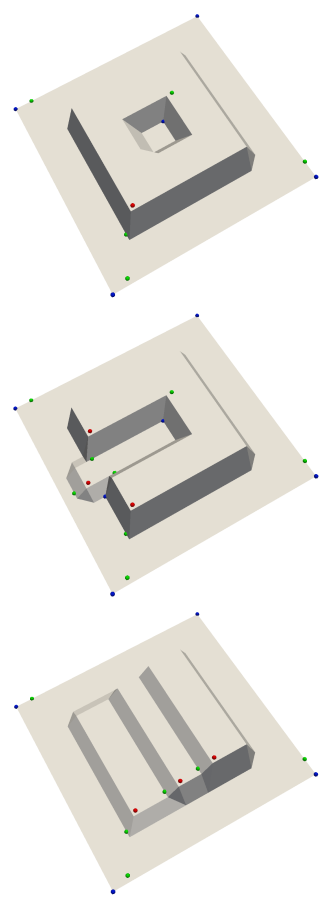

(b)
Figure 9: Our algorithm finds the correct number of critical simplexes, ${ }^{66}$ and (b) the one in [16] finds up to 5.6 times more critical simplexes. Dots mark maxima (red), minima (blue), saddles (green).

up to 2.1 times faster. The algorithm in [16], however, is dimension independent, while our algorithm is designed for triangle meshes only, and does not extend to higher dimensions in a straightforward manner. Another approach could be to eliminate plateaus with the method in [28] and then compute a discrete Morse gradient with the algorithm in [16]. However, the results presented in Table 1 make such a comparison unnececessary, because 677 our method is already faster than [16] alone.

\section{Concluding Remarks}

We have proposed a new algorithm that constructs a discrete Morse gradient on triangulated surfaces en- 685 dowed with a scalar field by starting from a watershed ${ }^{686}$ decomposition of their set of vertices. The first property ${ }_{688}^{687}$ of our approach is that the discrete Morse gradient com- 689 puted maintains the vertex classification inferred by the ${ }^{690}$ watershed decomposition, since the vertex-edge pairs ${ }_{692}^{691}$ are built in the same way as in the watershed decom- 693 position. By combining an algorithm for watershed de- 694 composition as a preprocessing step and the algorithm ${ }^{695}$ proposed here, we are able to process scalar fields that ${ }_{696}^{696}$ are not generic, i.e., that have adjacent vertices with 698 same function value. This opens up to the use of discrete Morse theory in real applications where flat areas are common, such as in terrain modeling but not only. Our method is preferable to other approaches based on simulation of simplicity [4, 22], since it preserves the original number of topological features. We have discussed the soundness of the new method and have demonstrated the importance of properly handling flat areas when dealing with morphological analysis of terrains and shapes. Also we have provided a fair comparison between our algorithm and the state of the art in computing a discrete Morse gradient starting from a given scalar field in 2D. We are currently working on a parallel implementation of our algorithm. In particular the vertex-edge pairing (section 5) and the edge-triangle pairing (section 6) can be parallelized improving time efficiency. We think that extending the computation of a discrete Morse gradient to functions with flat areas will strongly encourage the wider use of topological tools in shape analysis. The source code is publicly available (https://github.com/IuricichF/FormanGradient2D).

\section{Acknowledgements}

All the datasets used are courtesy of the Aim@Shape repository [30] and the Virtual Terrain Project [31]. This work has been partially supported by the US National Science Foundation under grant number IIS-1116747. The support of the Hungarian Academy of Sciences through the DOMUS project is also acknowledged.

\section{References}

[1] Forman, R.. Morse Theory for Cell Complexes. Advances in Mathematics 1998;134:90-145.

[2] Milnor, J.. Morse Theory. Princeton University Press, New Jersey; 1963. ISBN 0-691-08008-9.

[3] De Floriani, L., Fugacci, U., Iuricich, F., Magillo, P.. Morse complexes for shape segmentation and homological analysis: discrete models and algorithms. Computer Graphics Forum 2015;34(2):761-785. doi $10.1111 /$ cgf.12596

[4] Edelsbrunner, H., Mücke, E.P.. Simulation of simplicity: A technique to cope with degenerate cases in geometric algorithms. ACM Trans Graph 1990;9(1):66-104. doi $10.1145 / 77635$ 77639

[5] Edelsbrunner, H., Letscher, D., Zomorodian, A.. Topological persistence and simplification. Discrete \& Computational Geometry 2002;28(4):511-533. doi $10.1007 /$ s00454-002-2885-2

[6] De Floriani, L., Iuricich, F., Magillo, P., Simari, P.. Image Analysis and Processing - ICIAP 2013: 17th International Conference, Naples, Italy, September 9-13, 2013, Proceedings, Part II; chap. Discrete Morse versus Watershed Decompositions of Tessellated Manifolds. Berlin, Heidelberg: Springer Berlin Heidelberg. ISBN 978-3-642-41184-7; 2013, p. 339-348. 


\begin{tabular}{|c|c|c|c|c|c|c|c|c|c|c|}
\hline \multirow{2}{*}{ Dataset } & \multirow{2}{*}{$|V|$} & \multirow{2}{*}{$|T|$} & \multirow{2}{*}{$|C P|$} & \multicolumn{4}{|c|}{ Our Method. } & SoS [4] & \multirow[t]{2}{*}{ SoS++ 22} & \multirow{2}{*}{$\begin{array}{r}\text { Exp. 16 } \\
\text { Time }\end{array}$} \\
\hline & & & & 1 & 2 & 3 & Tot. & $\mid \mathrm{Cl}$ & & \\
\hline Crater & $100 \mathrm{~K}$ & $199 \mathrm{~K}$ & 869 & 0.4 & 0.4 & 0.3 & 1.3 & $1637_{(+46.9 \%)}$ & $16099_{(+45.9 \%)}$ & $2.6(\mathrm{x} 2.00)$ \\
\hline GardaLake & $810 \mathrm{~K}$ & $1.6 \mathrm{M}$ & 62291 & 2.9 & 2.9 & 2.9 & 10.1 & $99259(+37.2 \%)$ & $77733(+19.8 \%)$ & $20.6(\times 2.03)$ \\
\hline Kilimanjaro & $490 \mathrm{~K}$ & $0.9 \mathrm{M}$ & 26175 & 1.5 & 1.7 & 1.5 & 5.4 & $38591(+32.1 \%)$ & $29297(+10.6 \%)$ & $11.6(\mathrm{x} 2.14)$ \\
\hline ComoLake & $810 \mathrm{~K}$ & $1.6 \mathrm{M}$ & 43269 & 2.3 & 2.8 & 2.6 & 8.9 & $68729(+37.0 \%)$ & $52573(+17.6 \%)$ & $19.3(\mathrm{x} 2.16)$ \\
\hline Baia & $4.1 \mathrm{M}$ & $8.3 \mathrm{M}$ & 31805 & 9.5 & 17.2 & 16.0 & 48.8 & $37541_{(+15.2 \%)}$ & $34665(+8.2 \%)$ & 91.8 (x1.88) \\
\hline Puget & $9 \mathrm{M}$ & $19 \mathrm{M}$ & 328235 & 26.7 & 39.5 & 37.2 & 122.1 & $417943(+21.4 \%)$ & $347649(+5.5 \%)$ & $218.9(\mathrm{x} 1.79)$ \\
\hline OilPump & $0.5 \mathrm{M}$ & $1.1 \mathrm{M}$ & 24436 & 1.3 & 2.1 & 0.9 & 5.3 & $28070_{(+13.0 \%)}$ & $26210_{(+6.7 \%)}$ & $13.1(\mathrm{x} 2.4)$ \\
\hline Carter & $0.5 \mathrm{M}$ & $1.0 \mathrm{M}$ & 35306 & 1.9 & 1.3 & 1.1 & 5.8 & $52336(+32.3 \%)$ & $47386_{(+25.4 \%)}$ & $12.4(\mathrm{x} 2.0)$ \\
\hline Eros & $0.4 \mathrm{M}$ & $0.9 \mathrm{M}$ & 41236 & 1.2 & 1.5 & 0.9 & 4.5 & $63182(+34.8 \%)$ & $45748(+9.8 \%)$ & $10.8(\mathrm{x} 2.4)$ \\
\hline Rim01 & $0.6 \mathrm{M}$ & $1.3 \mathrm{M}$ & 57320 & 3.0 & 2.2 & 2.0 & 8.4 & $83722(+31.6 \%)$ & $75026(+23.5 \%)$ & $14.9(\mathrm{x} 1.7)$ \\
\hline Neptune & $0.2 \mathrm{M}$ & $4.0 \mathrm{M}$ & 272546 & 7.2 & 6.6 & 5.4 & 23.9 & $390988(+30.3 \%)$ & $334558(+18.5 \%)$ & $50.3(\times 2.1)$ \\
\hline
\end{tabular}

Table 1: Results obtained from eleven triangle meshes. The first six represent terrain datasets, where the scalar function is elevation. The last five are freeform surfaces, and the scalar function defined on them is mean curvature. The columns show, for each mesh, the number of vertices, triangles, critical simplexes, the computation times for our algorithm for performing steps 1,2 and 3, and the total computation time of our approach including the preprocessing step computing the input watershed segmentation, the number of critical simplexes obtained while applying simulation of simplicity (SoS) and the improved version described in [22] (SoS++). The last column shows the computational time for the homotopy expansion algorithm (timings are expressed in seconds). Blue and red values indicate the lowest and highest value for each column, respectively.

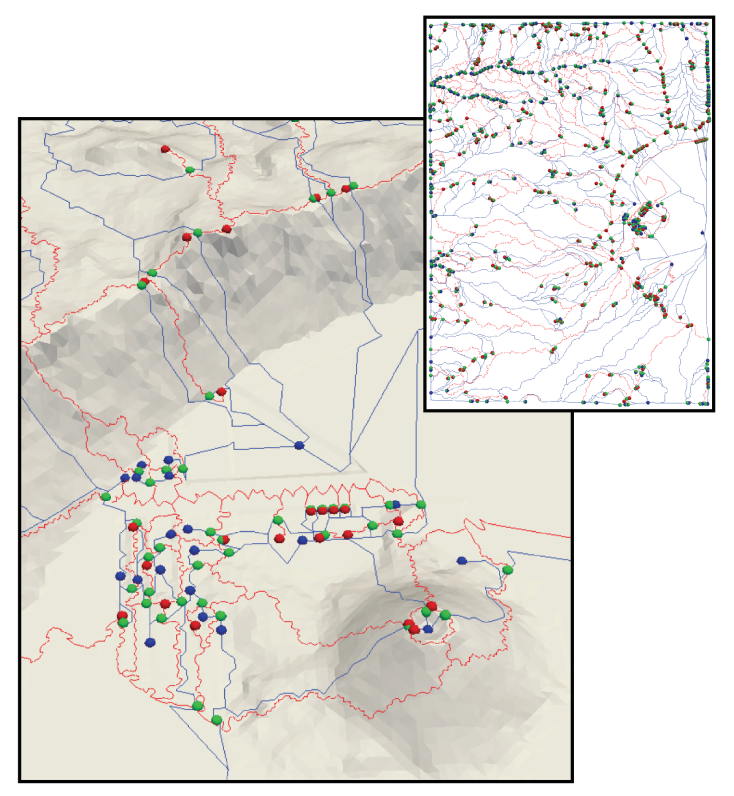

(a)

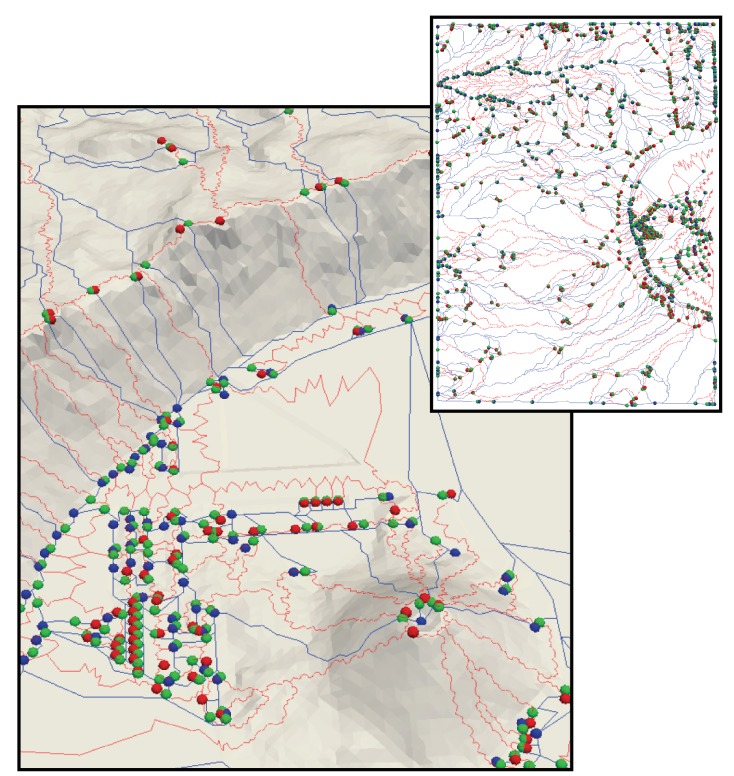

(b)

Figure 10: The 953 critical simplexes, connected through a discrete Morse gradient, computed on the Crater dataset (a) using our algorithm. Using the algorithm in [16] and SoS 1761 critical points are found (b). Red, blue and green dots mark maxima, minima and saddles, respectively. 


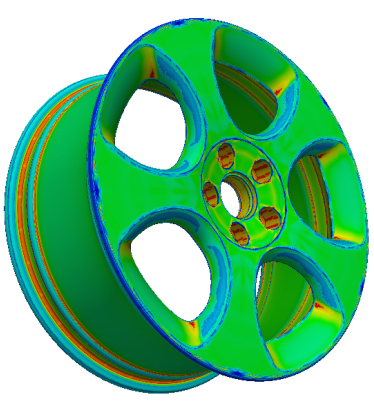

(a)

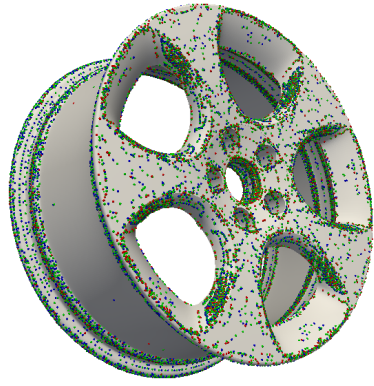

(b)

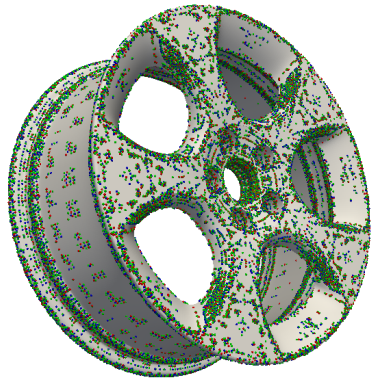

(c)

Figure 11: (a) The Rim01 dataset enriched with the mean curvature for each vertex. Our method identifies 57320 critical points (a) while using the algorithm in [16] and SoS, 83722 critical points are found (b). Red, blue and green dots mark maxima, minima and saddles, respectively.

[7] Meyer, F.. Topographic distance and watershed lines. Signal Pro- 746 cessing 1994;38:113-125. doi $10.1016 / 0165-1684(94) 747$ 90060-4

[8] Najman, L. Schmitt, M. Watershed of continuous functions. 749 Signal Processing 1994;38(1):99-112.

[9] Forman, R.. Combinatorial vector fields and dynamical systems, 751 Mathematische Zeitschrift 1998;228:629-681. doi 10.1007/ 752 PL00004638

[10] Forman, R.. A user's guide to discrete Morse theory. Séminaire 754 Lotharingien de Combinatoire 2002;48:35.

[11] Vincent, L., Soille, P.. Watershed in digital spaces: An effi- 756 cient algorithm based on immersion simulation. IEEE Trans on 757 Pattern Analysis and Machine Intelligence 1991;13(6):583-598. 758 doi $10.1109 / 34.87344$

12] Soille, P.. Morphological Image Analysis: Principles and Ap- 760 plications. Springer-Verlag, Berlin and New York; 2004. ISBN 761 3540429883 .

[13] Roerdink, J., Meijster, A.. The watershed transform: Defi- 763 nitions, algorithms, and parallelization strategies. Fundamenta 764 Informaticae 2000;41:187-228.

[14] Stoev, S.L., Strasser, W.. Extracting regions of interest apply- 766 ing a local watershed transformation. In: Proc. IEEE Visualiza- 767 tion'00. ACM Press. ISBN 1-58113-309-X; 2000, p. 21-28. 768

[15] Mangan, A., Whitaker, R.. Partitioning 3D surface meshes 769 using watershed segmentation. Trans on Visualization and 770 Computer Graphics 1999;5(4):308-321. doi:10.1109/2945. 771 817348

[16] Robins, V., Wood, P.J., Sheppard, A.P.. Theory and algorithms 773 for constructing discrete Morse complexes from grayscale digi- 774 tal images. IEEE Trans on Pattern Analysis and Machine Intel- 775 ligence 2011;33(8):1646-1658. doi 10.1109/TPAMI.2011. 776 95

[17] Shivashankar, N., Maadasamy, S., Natarajan, V.. Parallel 778 computation of 2D Morse-Smale complexes. IEEE Trans on 779 Visualization and Computer Graphics 2012;18(10):1757-1770. 780 doi $10.1109 /$ TVCG.2011.284

18] Shivashankar, N., Natarajan, V.. Parallel computation 782 of 3D Morse-Smale complexes. Computer Graphics Forum 783 2012;31(3):965-974. doi $10.1111 / j .1467-8659.2012 .784$ $03089 . x$

[19] Weiss, K., Iuricich, F., Fellegara, R., De Floriani, L.. A pri- 786 $\mathrm{mal} / \mathrm{dual}$ representation for discrete Morse complexes on tetra- 787 hedral meshes. Computer Graphics Forum 2013;32(3):361-370. doi $10.1111 / \mathrm{cgf} .12123$

[20] Gyulassy, A., Bremer, P., Pascucci, V.. Computing MorseSmale complexes with accurate geometry. IEEE Trans Vis Comput Graph 2012;18(12):2014-2022. doi 10 .1109/TVCG.
2012.209

[21] King, H., Knudson, K., Mramor, N.. Generating discrete Morse functions from point data. Experimental Mathematics 2005;14(4):435-444. doi http: //projecteuclid.org/ euclid.em/1136926974

[22] Gyulassy, A., Bremer, P.T., Hamann, B., Pascucci, V.. Practical considerations in Morse-Smale complex computation. In: Topological Methods in Data Analysis and Visualization: Theory, Algorithms, and Applications. Mathematics and Visualization; Heidelberg: Springer Verlag; 2011, p. 67-78. doi 10 1007/978-3-642-15014-2_6

[23] Fellegara, R., Iuricich, F., De Floriani, L., Weiss, K.. Efficient computation and simplification of discrete Morse decompositions on triangulated terrains. In: SIGSPATIAL'14 Proc. 22nd Int. Conf. on Advances in Geographic Information Systems. 2014, p. 223-232. doi 10.1145/2666310.2666412

[24] Günther, D., Reininghaus, J., Wagner, H., Hotz, I. Efficient computation of 3D Morse-Smale complexes and persistent homology using discrete Morse theory. The Visual Computer 2012;28(10):959-969. doi 10.1007/ s00371-012-0726-8

[25] Gyulassy, A., Günther, D., Levine, J.A., Tierny, J., Pascucci, V.. Conforming Morse-Smale complexes. IEEE Trans Vis Comput Graph 2014;20(12):2595-2603. doi 10 .1109/TVCG. 2014.2346434

[26] Meyer, F., Beucher, S.. Morphological segmentation. J of Visual Communication and Image Representation 1990;1:21-45. doi 10.1016/1047-3203(90)90014-M

[27] Dijkstra, E.W.. A note on two problems in connexion with graphs. Numerische Mathematik 1959;1:269-271. doi 10 $1007 / \mathrm{BF} 01386390$

[28] Magillo, P., De Floriani, L., Iuricich, F.. Morphologicallyaware elimination of flat edges from a TIN. In: Proc. 21th ACM SIGSPATIAL Int. Conf. on Advances in Geographic Information Systems. 2013, p. 244-253. doi $10.1145 / 2525314$ 2525341

[29] Banchoff, T.. Critical points and curvature for embedded polyhedral surfaces. American Mathematical Monthly 1970;77(5):475485.

[30] Aim@shape. http://visionair.ge.imati.cnr.it/ontologies/shapes/; 2016.

[31] Virtual Terrain Project. http://vterrain.org; 2016 


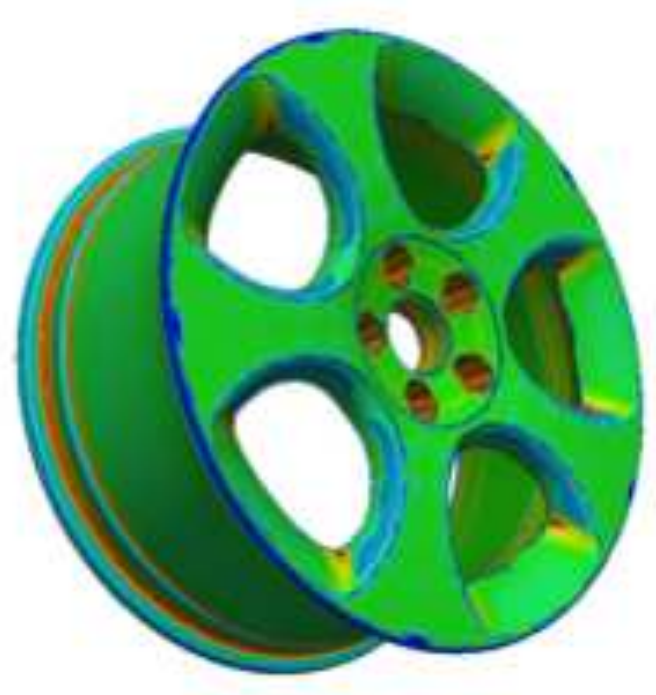

Original scalar field

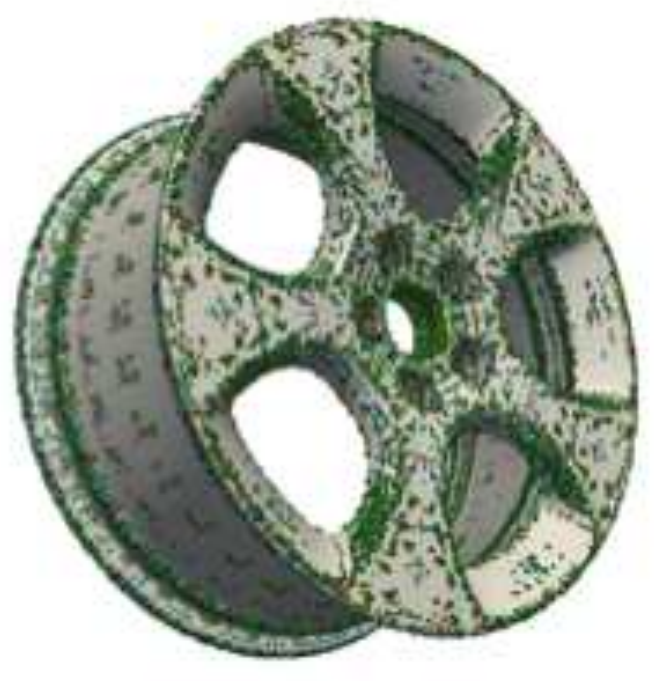

Simulation of Simplicity

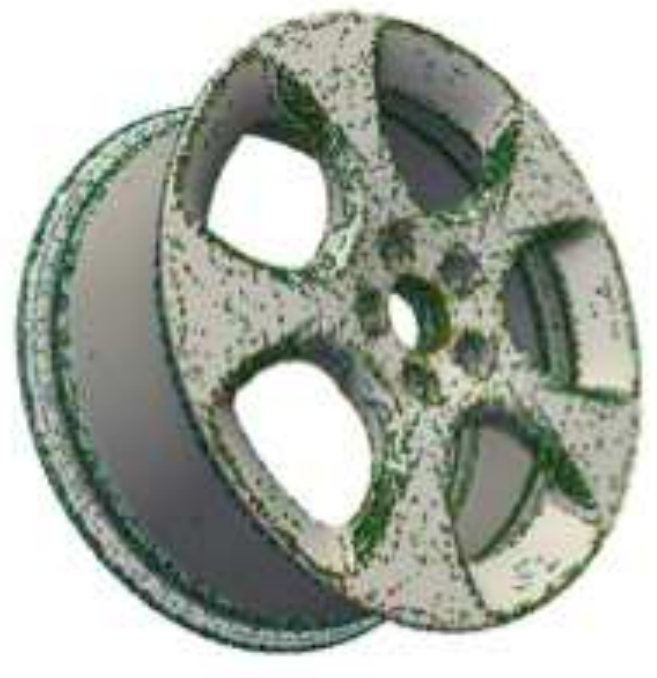

Our method 